\title{
Research on Pinch Plasma Focus Devices of Hundred of Kilojoules to Tens of Joules
}

\author{
Leopoldo Soto, Patricio Silva, José Moreno, Gustavo Silvester, Marcelo Zambra, Cristian Pavez ${ }^{1}$ \\ Luis Altamirano ${ }^{2}$, Horacio Bruzzone ${ }^{3}$, Mario Barbaglia ${ }^{4}$, Yurii Sidelnikov ${ }^{5}$ and Walter Kies ${ }^{6}$ \\ Comisión Chilena de Energía Nuclear, Casilla 188-D, Santiago, Chile \\ ${ }^{1}$ Universidad de Concepción, Chile \\ ${ }^{2}$ Dicontek, Chile \\ ${ }^{3}$ Universidad Nacional de Mar del Plata and CONICET-PLADEMA, Argentina \\ ${ }^{4}$ PLADEMA-Universidad Nacional del Centro, Tandil, Argentina \\ ${ }^{5}$ Institute of Spectroscopy, RAS, Troitsk, Moscow Region, Russia \\ ${ }^{6}$ Heinrich-Heine-Universität, Düsseldorf, Germany
}

Received on 26 February, 2004; revised version received on 24 May, 2004

\begin{abstract}
At present the Plasma Physics and Plasma Technology Group of the Comisión Chilena de Energía Nuclear (CCHEN) has the experimental facilities in order to study fast dense transient discharges in a wide range of energy and current, namely: I) energy from hundred of kilojoules to tens of joules, II) current from megaamperes to tens of kiloamperes. Also several diagnostics have been implemented. An overview of the work being carried out on dense pinch plasma focus discharges at the Comisión Chilena de Energía Nuclear is presented. The plasma energy density and scaling laws for the neutron yield are discussed. Possible applications of the radiation emitted are also discussed.
\end{abstract}

\section{Introduction}

Pinch plasmas can produce high energy density conditions, instability phenomena, plasma jets, $\mathrm{X}$-ray and neutron emission. Many of the observed phenomena in pinch plasmas are not completely understood. At present the Plasma Physics and Plasma Technology Group of the Comisión Chilena de Energía Nuclear (CCHEN) has the experimental facilities in order to study dense transient discharges in a wide range of energy and current keeping the same time scale. The program research includes: a) mechanism of X-ray emission (thermal vs. beam bremsstrahlung), population inversion in soft X-ray to VUV region, mechanism of neutron emission (thermonuclear vs. beam-target), charged particles beams emission; b) development of diagnostics; and c) development of optimized apparatus for flash sources of neutrons and x-rays (nanoflashes) and possible applications.

The devices at CCHEN are: a small and fast capillary discharge $(1-2 n F, 5 k A,<1 J, 5 n s$ time to peak current, $\left.d I / d t \sim 10^{12} A / s\right)$; two very small plasma focus devices, PF-50J (160nF, 25kV,60kA, 50J, 150ns time to peak current, $\left.d I / d t \sim 3 \cdot 10^{11} A / s\right)$ and PF-400J $(880 \mathrm{nF}, 30 \mathrm{kV}, 120 \mathrm{kA}, 400 \mathrm{~J}, 300 \mathrm{~ns}$ time to peak current, $\left.d I / d t \sim 4 \cdot 10^{11} A / s\right) ;$ a small plasma focus SPEED4 $(1.25 \mathrm{mF}, 100 \mathrm{kV}, 550 \mathrm{kA}, 6.25 \mathrm{~kJ}, 350 \mathrm{~ns}$ time to peak current, $\left.d I / d t \sim 10^{12} \mathrm{~A} / \mathrm{s}\right)$; and the pulse power generator SPEED2, a medium energy and large current device $(4.1 \mu \mathrm{F}$, $300 k V, 4 M A, 187 k J, 400 n s$ time to peak current, $d I / d t \sim$ $10^{13} \mathrm{~A} / \mathrm{s}$ ). The SPEED2 arrived at the CCHEN in May 2001 from Düsseldorf University, Germany, and it is in operation since January 2002, being the most powerful and energetic device for dense transient plasma in the Southern Hemisphere.

Several diagnostics have been implemented: voltage, total current and current derivative monitors; plasma images with an intensified CCD camera gated at 5 ns exposure time; silver activation counter and ${ }^{3} \mathrm{He}$ detectors for neutron yield measurements; plastic scintillator with photomultiplier for $\mathrm{X}$-ray and neutron detection with temporal resolution; VUV and soft X-ray spectroscopy; and pulsed optical refractive diagnostics using a pulsed Nd-YAG laser.

In this article we present results related to our plasma focus research. A plasma focus is a particular pinch discharge in which a high pulsed voltage is applied to a low pressure gas between coaxial cylindrical electrodes. The central electrode is the anode partially covered with a coaxial insulator. The discharge starts over the insulator surface, and afterwards the current sheath is magnetically accelerated along the coaxial electrodes. After the current sheath runs over the ends of the electrodes the plasma is compressed in a small cylindrical column (focus or pinch). Fig. 1 shows a scheme of the circuit and the plasma dynamics. The electrodes are in vertical positions, the anode in the center is partially covered from its base by a coaxial insulator. In most of the devices these three stages last a few microseconds. The maximum pinch compression should be coincident with peak current in order to achieve the best efficiency. The pinch generates beams of ions and electrons, 
and ultra-short X-ray pulses. In the pinch the temperature is $\sim 1 \mathrm{keV}$ and the density is $\sim 10^{25} \mathrm{~m}^{-3}$. Using deuterium gas, plasma focus devices produce fusion D-D reactions, generating fast-neutrons pulses $(\sim 2.5 \mathrm{MeV})$ and protons (leaving behind ${ }^{3} \mathrm{He}$ and ${ }^{3} \mathrm{H}$ ). The neutrons burst usually lasts about tens to hundreds of nanoseconds. The emitted neutrons can be applied to perform radiographs and substance analysis, taking advantage of the penetration and activation properties of this neutral radiation. The plasma focus is a pulsed neutron source especially suited for applications because it reduces the danger of contamination of conventional isotopic radioactive sources. A passive radioactive source of fast neutrons with similar energy (for instance ${ }^{252} \mathrm{Cf}$ with similar mean energy or Am/Be with a harder spectrum) emits continuously, causing inconveniences in handling and storing. In turn, plasma-focus generators do not have activation problems for storage and handling.

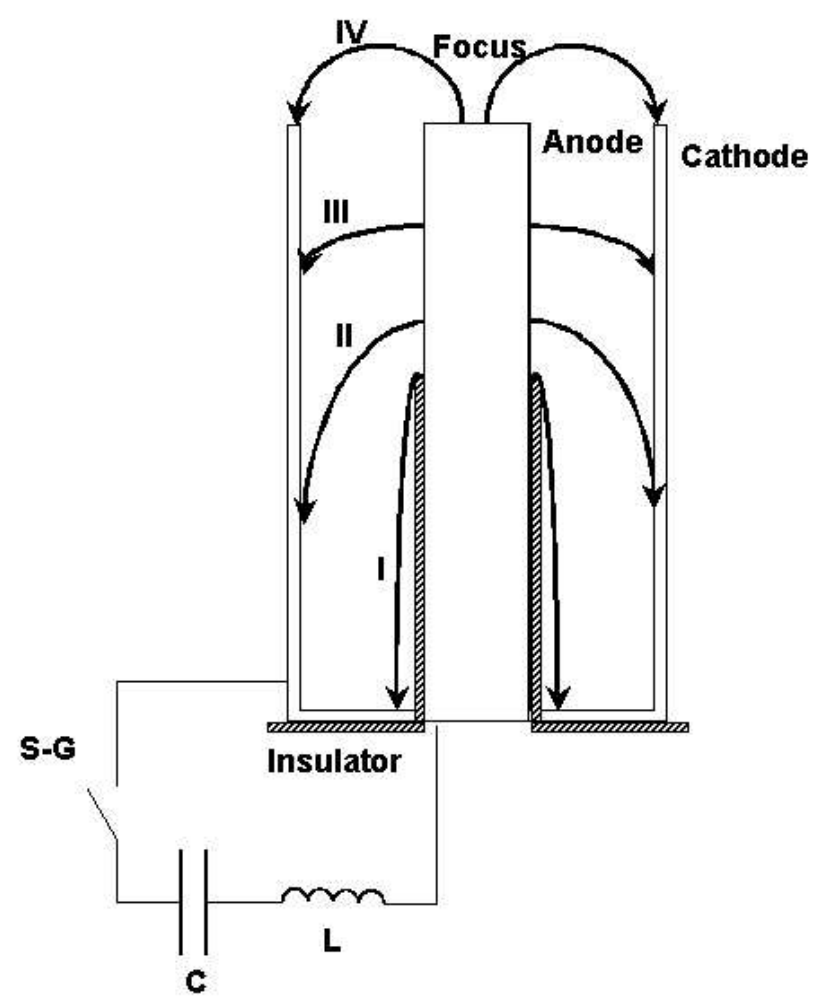

Figure 1. A scheme of the circuit and the plasma dynamics is shown. The capacitor $\mathrm{C}$ is discharged over the electrode through a spark gap (SG). The plasma dynamics is sketched in a side section of the electrodes, I: discharge starts over the insulator, II, III: the current sheath is accelerated along the coaxial electrodes, and IV: pinch.

During the last thirty years, substantial efforts and resources have been invested in the study of X-ray and neutrons emitted by plasma focus devices [1-5]. The studies range from small devices around hundreds of Joules, to large facilities about $1 M J$. Specifically in the $3 k J$ range $[4,5]$ there are numerous results obtained by the AsianAfrican Association for Plasma Training Network (AAAPT). Plasma-focus neutronic emissions have been found ranging from $10^{7}$ neutrons with $1 \mathrm{~kJ}$ drivers, up to $10^{12}$ neutrons with $1000 \mathrm{~kJ}$. Had small portable PF devices were available, the value of the emissions would be substantially increased, for a number of nuclear techniques could be produced in wider domains of applications. There are few published works about devices designed to operate at hundred of joules $[6,7]$ and they operate with slow drivers $(\sim 10 \mu \mathrm{F}$ capacitor bank, $\sim 100-70 \mathrm{nH}, \sim 7 \mathrm{kV}, \sim 250 \mathrm{~J}, \sim 1.3 \mu \mathrm{s}$ current rise time).

In spite of all the accumulated research related to plasma foci, there are several questions still waiting for answers, particularly those concerning the sheath formation, insulator conditioning and influence of gas impurities. An area of research that is not well explored is that of the very-lowenergy plasma foci. Most of the experimental studies were focused in medium and large facilities from tens to hundreds of $\mathrm{kJ}$, or small devices about some kJ. In fact, we can question if good focussing can be achieved below $1 \mathrm{~kJ}$, and if so which are the appropriate design criteria and scaling laws in this energy region. Experimental research with a plasma focus driven by a capacitor bank of tens to hundred of joules would allow to extend the theoretical models to the region of low energy [8-10]. Moreover, a capacitor bank under the kilojoule has a small size in comparison with banks in the kilojoules range, thus it would be easier to operate in a repetitive regime from $\mathrm{Hz}$ to $\mathrm{kHz}$, since the power requirements and the spark-gap erosion are consequently lower. A review of our research in plasma focus devices is presented in this article. The design and construction of very small plasma foci (PF-50J and PF-400J); development of diagnostics and instrumentation (plasma dynamics observations and low fluence neutron detection); plasma dynamics and neutron emission from PF-50J and PF-400J; and preliminary results in SPEED2 operated in plasma focus mode are presented and discussed.

\section{Compact and fast plasma foci of tens and hundred of joules: PF-50J and PF-400J}

An area of research that is not well explored is that of the very-small low-energy plasma foci. Feasibility objections have been made to devices with lower energies (less than $1 k J)$, for not having enough energy and time to create, move and compress the plasma. We have shown that those objections are not applicable [11, 26, 27].

As first stage of a program to design a repetitive pulsed radiation generator for industrial applications we constructed two very small plasma focus operating at an energy level of the order of a) tens of joules (PF-50J, 160nF capacitor bank, $20-35 k \mathrm{~V}, 32-100 \mathrm{~J}, \sim 150 \mathrm{~ns}$ time to peak current) [8-10] and b) hundred of joules (PF-400J, 880 $\mathrm{nF}$, $20-35 k \mathrm{~V}, 176-539 \mathrm{~J}, \sim 300 n s$ time to peak current) [11].

2.1 Design criteria. The compact and fast plasma foci devices constructed by CCHEN, PF-400J and PF-50J, consists of capacitors banks that discharge over coaxial electrodes through spark-gaps. The capacitor bank consists in four capacitors connected in parallel. Four capacitors of 
$220 n F-20 n H$ in PF-400J case and $40 n F-20 n H$ in PF-50J case. The devices operate with charging voltages of 20 to $35 \mathrm{kV}$. In order to obtain low inductance the capacitors were connected in a compact layout. Short and coaxial spark-gaps were designed for the same purpose. The measured total external inductance is $38 n H$. The total impedance of the generator is of the order of $0.2 \Omega$ for the PF$400 \mathrm{~J}$ and $0.5 \Omega$ for the PF-50J. To determine the size of the electrodes the design relations suggested by S. Lee [4] and a theoretical model of plasma focus for neutron production [12] were applied. It is known that the pinch phase in a plasma focus is highly dependent of the current sheath formation over the insulator. Unfortunately, there are still not validated theoretical models to determine the dimensions of the insulator. Therefore, several tests with different insulator length and diameter, scanning pressure range from 1 to 12 mbar, were necessary to determine the size of the insulator in order to obtain a homogeneous initial sheath. The current sheath was studied with an image converter camera with $5 \mathrm{~ns}$ exposure time. The final electrodes for the PF$400 \mathrm{~J}$ consist of a $28 \mathrm{~mm}$ long, $12 \mathrm{~mm}$ diameter cooper tube anode, and an outer cathode of eight $5 \mathrm{~mm}$ diameter copper rods uniformly spaced on a $31 \mathrm{~mm}$ diameter circumference. The anode and cathode were separated by an alumina tube of $21 \mathrm{~mm}$ length. In the case of the PF-50J after some improvements from the original design $[9,10,26]$, the electrodes configuration consists of a $29 \mathrm{~mm}$ long, $6 \mathrm{~mm}$ diameter copper tube anode, and an outer cathode of eight, $5 \mathrm{~mm}$ diameter cooper rods uniformly spaced on a circumference with diameter of $27 \mathrm{~mm}$. Anode and cathode were separated by an alumina tube of $24 \mathrm{~mm}$ length. Such configurations resulted from the short first quarter period of the discharge current (200-300ns, due to the small bank capacity), which require a short effective anode $(6-7 \mathrm{~mm})$. The size of these devices is of the order of $25 \mathrm{~cm} \times 25 \mathrm{~cm} \times 50 \mathrm{~cm}$. The design calculations indicate that neutrons yields of $10^{4}-10^{5}$ neutrons per shot are expected with discharges in deuterium in the PF-50J and $5 \cdot 10^{5}-5 \cdot 10^{6}$ neutrons per shot in the PF-400J.

2.2 Electrical signals. Voltage, total current and current derivative are measured with usual monitors, a fast resistive divider, and a Rogowskii coil. The voltage monitor was located close to the plasma load. The Rogowskii coil monitored the current derivative signal in the capacitor bank.

2.3 Images from the visible plasma light. An intensified CCD camera (ICCD) gated at $5 n s$ exposure time, and synchronized with the discharge has been used in order to obtain side view images of the visible light emitted from the plasma [10]. For imaging the plasma over the microchannel plate in the ICCD camera, a regular bi-convex lens with a focal length of $12.5 \mathrm{~cm}$ and $5 \mathrm{~cm}$ of diameter was used. In order to increase the field depth a mask with an open circle of $1 \mathrm{~cm}$ diameter was attached to the lens, so an optical number $F=1 / 12.5$ was obtained. A magnification $m=0.2$ was used. The resolution of the camera for magnification $m=1$ is $23 \mu m$, thus for $m=0.2$ a resolution of $95 \mu m$ $(\sim 0.1 \mathrm{~mm})$ is achieved.

2.4 Neutron detection. A silver activation counter, calibrated with an Am-Be source, placed at $30 \mathrm{~cm}$ in the side- on direction was used to record the integrated neutron signal for total yield over $5 \cdot 10^{5}$ neutrons per shot (PF-400J). For total neutron yield lower than $5 \cdot 10^{5}$ neutrons per shot (PF-50J) a system based on ${ }^{3} \mathrm{He}$ detector was developed [13]. A conventional neutron detection technique was adapted to measure low neutron yields from D-D fusion pulses. ${ }^{3} \mathrm{He}$ proportional counters are well known neutron detectors whose detection principle is based on the nuclear reaction, $\mathrm{n}\left({ }^{3} \mathrm{He},{ }^{3} \mathrm{H}\right) \mathrm{p} \mathrm{[20]}$. An analogic signal corresponding to the current generated in the ${ }^{3} \mathrm{He}$ tube is registered through a preamplifier whose output is directly connected to a digital oscilloscope. The time-integrated signal is the charge generated in the ${ }^{3} \mathrm{He}$ tube and it is proportional to the neutron yield. Time of integration is determined by the characteristics of the preamplifier and is about some hundred of microseconds. No neutron background is detected during this temporal window. To calibrate the ${ }^{3} \mathrm{He}$ detection system (with moderator included) a silver activation counter (previously calibrated with an Am-Be source) was used as a neutron calibration reference. Both detectors, the adapted ${ }^{3} \mathrm{He}$ and the silver activation counter were used simultaneously in the PF-400J detecting neutron yields from $5 \times 10^{5}$ to $2 \times 10^{6}$ neutrons per shot. A linear proportional relation was obtained between the ${ }^{3} \mathrm{He}$ time integrated signal and the neutron yield measured by the silver activation counter. Neutron yields lower than $10^{3}$ neutrons per pulse were possible to be measured with this technique [13].

Also detectors based on plastic scintillator connected to photomultiplier for X-ray and neutron detection with temporal resolution have been implemented.

2.5 Results. In order to study the plasma motion images in the visible region were obtained with a $5 n s$ gated ICCD camera for different times of the discharge and for different Hydrogen pressures. A sequence of the plasma images of the PF-50J is presented in Fig. 2.

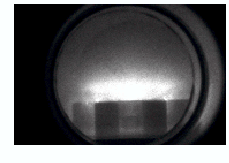

37ns

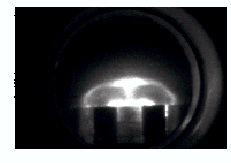

222ns

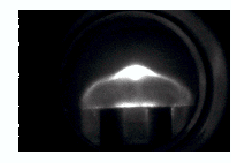

309ns
Figure 2. Sequence of the plasma images obtained with a ICCD camera gated at $5 n s$ of exposure time from different discharges in hydrogen at 0.47 mbar in the PF-50J [10].

The umbrella-like current sheath running over the end of the coaxial electrodes and the pinch after the radial collapse can be clearly observed in the photographs. Radial collapses of the order of $10^{5} \mathrm{~m} / \mathrm{s}$ were observed [10]. The first frame (37ns) suggests that there is not a good current sheath formation over the insulator. Several tests and improvements were necessary to determinate the dimensions of the insulator (see section "Design criteria") [11,26].

After some improvement from the original design, neutron emission studies were performed in discharges in deuterium at different pressures, 5 to $12 \mathrm{mbar}$, with a charging voltage of $30 \pm 2 k V$ in the PF-400J and $29 \pm 2 k V-25 \pm 2 k V$ in the PF-50J ( $\sim 400 J$ and $\sim 70-50 J$ stored in the capacitor bank respectively). 


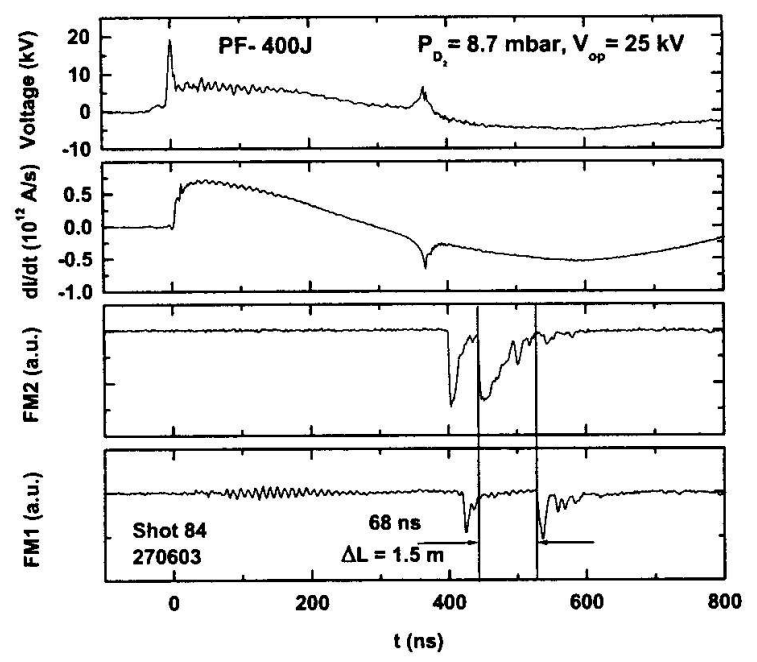

Figure 3. Electrical traces for a shot in deuterium at some mbar pressure. Peak currents of $127 \pm 6 k A$ and $60 \pm 3 k A$ were obtained for the PF-400J (8.7mbar) and PF-50J (7mbar). Also signals with scintillator with photomultiplier (FM1 and FM2) are shown for the PF-400J. The distance $\Delta$, between FM1 and FM2 was $\Delta=1.5 \mathrm{~m}$ and the time of flight for the neutrons was $\sim 65 \mathrm{~ns}$, thus $\sim 2.77 \mathrm{MeV}$ for the energy of the neutrons.
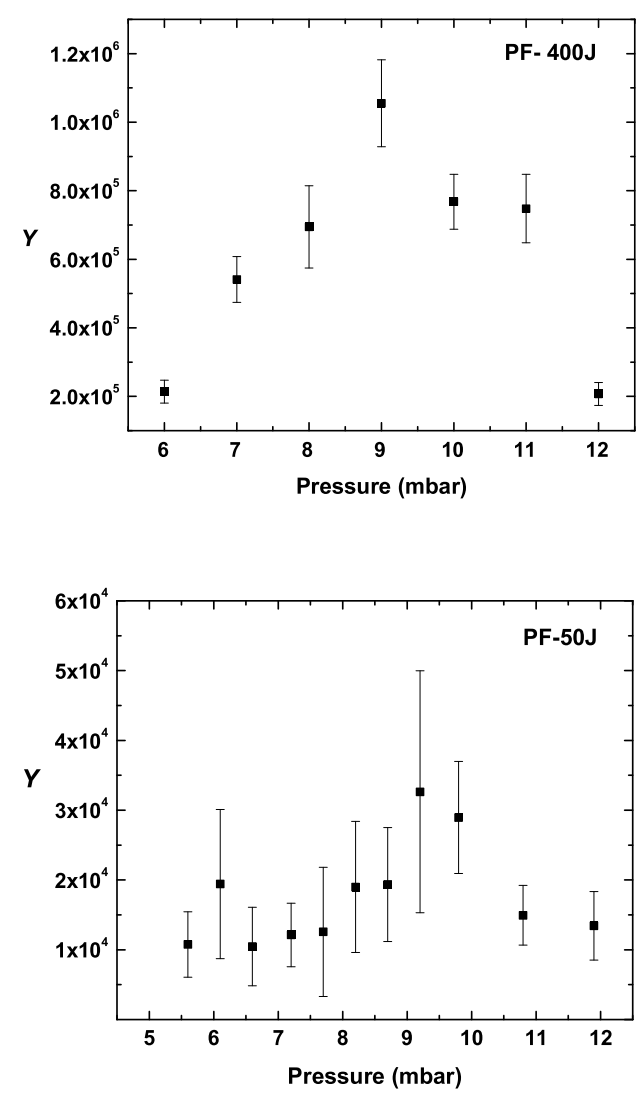

Figure 4. Neutron yield as a function of the filling gas pressure for the PF-400J (operated at $\sim 400 J$ ) [11] and PF-50J (operated at $\sim 70 J)$ [27].

Electrical traces for a shot in deuterium at some mbar pressure is shown in Fig. 3 where $127 \pm 6 k A$ and $60 \pm 3 k A$ peak current is obtained for the PF-400J and PF-50J. The typical dip in the signal of the current derivative associated with the formation of a pinched plasma column on the axis was observed. From the current derivative signals the implosion time (pinch time, measured at the moment for the minimum in $d I / d t$ ) versus filling pressure was obtained. The maximum compression of the plasma occurs close to the peak current for a pressure close to $7 m b a r$ in the PF-400J and close to $6 \mathrm{mbar}$ in the PF-50J.). Also signals from detectors based on plastic scintillator connected to photomultiplier (FM1 and FM2) are shown for the PF-400J. The distance $\Delta L$, between FM1 and FM2 was $\Delta L=1.5 \mathrm{~m}$ and the time of flight for the neutrons was $\sim 65 \mathrm{~ns}$, thus an energy of the order of $\sim 2.77 \mathrm{MeV}$ is estimated for the neutrons.

The neutron yield as a function of the filling gas pressure is shown in Fig. 4. Each point is the average of ten shots and the error bars are the standard deviations. The maximum measured neutron yield was $(1.06 \pm 0.13) \cdot 10^{6}$ neutrons per shot at 9 mbar in the PF-400J and $(3 \pm 1.5) \cdot 10^{4}$ neutrons per shot at 9 mbar in the PF-50J operating at $70 \mathrm{~J}$ and $(1.1 \pm 0.5) \cdot 10^{4}$ neutrons per shot at 6 mbar in the PF-50J operating at $50 \mathrm{~J}[27]$.

\section{SPEED2 AND SPEED4 Generators}

SPEED2 and SPEED4 are generators based on Marx technology and were designed in the University of Düsseldorf. SPEED2 consists on $40+/-$ Marx modules connected in parallel. Each module has 6 capacitors $(50 k \mathrm{~V}, 0.625 \mu \mathrm{F}$, $20 n H)$ and 3 sparkgaps, so the pulse power generator SPEED2 is a medium energy and large current device $(4.1 \mu \mathrm{F}$ equivalent Marx generator capacity, $300 \mathrm{kV}, 4 \mathrm{MA}$ in short circuit, $187 \mathrm{~kJ}, 400 \mathrm{~ns}$ rise time, $d I / d t \sim 10^{13} \mathrm{~A} / \mathrm{s}$ ) [2].The SPEED2 arrived at the CCHEN in May 2001 from Dsseldorf University, Germany, and it is in operation since January 2002, being the most powerful and energetic device for dense transient plasma in the Southern Hemisphere. Moreover, SPEED2 is the unique dense plasma transient experiment operating at currents of Mega-amperes in Chile. Simultaneously an intermediate device, SPEED4, has been constructed. It consists of $4+/-$ Marx modules connected in parallel, each module has 2 capacitors and 1 sparkgap $(50 \mathrm{kV}, 0.625 \mu \mathrm{F}, 20 \mathrm{nH})$, and currently is being set up in operation (SPEED4: $1.25 \mu F$ equivalent Marx generator capacity, $100 \mathrm{kV}, 550 \mathrm{kA}, 350 \mathrm{~ns}$ rise time, $d I / d t \sim$ $10^{12} \mathrm{~A} / \mathrm{s}$ ).

Experiments in different Z-pinch configurations, at current of hundred of kiloamperes to mega-amperes, using the SPEED 2 generator will be carried out. A device designed 15 years ago will be used for the research of scientific topics relevant today and for the research and development of new ideas.

Most of the previous works developed in SPEED2 at Düsseldorf were done in a plasma focus configuration for $\mathrm{X}$-ray emission and the neutron emission from SPEED2 was not completely studied. The Chilean operation has begun implementing and developing diagnostics in a conventional plasma focus configuration in order to characterized the neutron emission. Then, after getting the experimental expertise 
with SPEED2, new experiments in a quasi-static Z-pinch [15-18], gas puffed plasma focus, and wires array will be developed to extend the device capabilities. Also SPEED2 and SPEED4 will be used in the development of applications of radiation pulses from hot, dense plasmas, including $\mathrm{X}$ ray and neutron radiography, detection of substances, microradiography for applications to microelectronic lithography and diagnostics of nanostructures. Plasma focus on SPEED 2 is an intense pulsed source of neutrons $\left(10^{11}-10^{12}\right.$ neutrons per pulse) and X-rays. Plasma focus in SPEED 4 with heavy gases will be used as an intense source of soft X-rays. The applications will be developed using the above generators in order to determine the radiation threshold for the various types of applications (radiography, neutrography, substances detection). This information will be used to design smaller devices suited for applications.

3.1 Preliminary results in SPEED2. The Chilean operation of the SPEED2 device has begun implementing and developing diagnostics in a conventional plasma focus configuration. Discharges in plasma focus mode have been performed at $+/-30 k V$ charging voltage in a (i.e. $180 k V, 67 k J)$. A peak current greater than $2 M A$ was achieved. Fig. 5 shows the corresponding electrical traces. Fig. 5 a) corresponds to a discharge at 5.9 mbar deuterium, where the load is practically the inductance of the central collector and electrodes $(\sim 5 n H)$. Fig. 5 b) corresponds to 2.5 mbar, where the pinch phenomenon is clearly observed in the peak voltage and in the dip of the $d I / d t$.

(a)

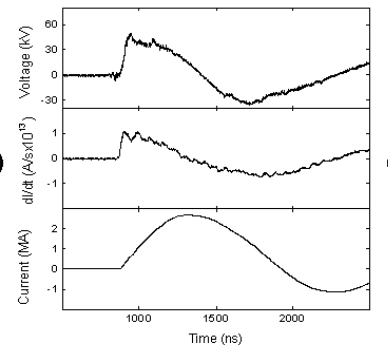

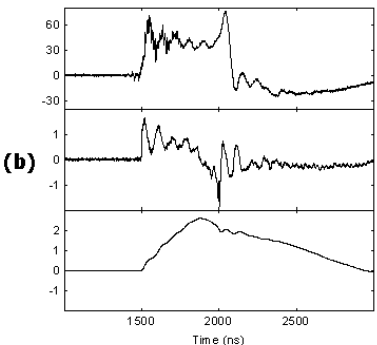

Figure 5. Electrical signals in SPEED2 generator operating in Chile at CCHEN. Discharges in plasma focus mode have been performed $( \pm 30 \mathrm{kV}$ charging voltage in a three step $+/-$ Marx generator, i.e. $180 \mathrm{kV}, 67 \mathrm{~kJ})$. A peak current greater than $2 \mathrm{MA}$ is achieved. a) 5.9 mbar $D_{2}$, b) 2.5 mbar $D_{2}$, at this pressure the pinch phenomena is clearly observed in the peak voltage and in the dip of the $d I / d t$.

SPEED2 uses a especial insulator, quartz covered with alumina, and it requires several shots of preparation in order to obtain a neutron yield with a dispersion lower than $30 \%$ between shots. We have not had enough shots with the same insulator in order to achieve the proper conditions of operation. Preliminary results obtained at CCHEN show a neutron yield of the order of $10^{10}-10^{11}$ neutrons per shot. In Düsseldorf a neutron yield of the order of $10^{11}-10^{12}$ neutrons per shot was obtained [28].

Time resolution neutron detection are now being implemented.

\section{Discussion and conclusions}

In the table 1 the principal parameters of the plasma focus devices at the Comisión Chilena de Energía Nuclear are listed. In table 2 the results related to the neutron yield obtained in the experiments presented in this article are shown.

Two very small plasma focus have been designed, constructed and set up in operation. One in the rage of hundred of joules (PF-400J) and other in the range of tens of joules (PF-50J). The region of tens joules was unexplored up to now. Neutron emission have been obtained in both devices. An especial technique was necessary to develop to detect neutron pulsed of the order of $10^{4}$ neutrons per shot. The measured neutron yield was of the order of $10^{6}$ and $10^{4}$ neutrons per shot in the PF-400J and PF-50J respectively (table 2). A repetitive neutron pulsed generator based on a very low energy plasma focus as those reported here could be a breakthrough in engineering applications such as soil humidity measurements, medical neutron therapies and substances detection (explosives, drugs, minerals, etc). According to commercial information, $10^{6}-10^{8}$ neutrons/s at $10 \mathrm{kHz}$ repetition rate are useful for prompt gamma neutron analysis. This translates into a $10^{2}-10^{4}$ neutrons/shot. A PF-50J device operating at $1 k H z$ could be used in such kind of applications. The devices presented here, conceived for laboratory purposes, are single shot machines that can be operated only at $0.5-1 \mathrm{~Hz}$.

A comparison between plasma foci of different energies is necessary. Although only fraction of the initial energy stored $E$ in the capacitor bank is transferred to the plasma, the parameter $E / V_{P}$ (with $V_{P}$ the plasma volume) is usually used to characterize the plasma energy density in order to compare different devices. According with scaling laws [20] and optical diagnostics [10] the final pinch radius (previous to appearance of instabilities with the subsequent appearance of smaller inhomogeneities in the plasma column) is of the order of $0.12 a$ and the maximum pinch length is of the order $0.8 a$. Thus the final plasma volume $V_{P}$ (previous to appearance of probable instabilities) is of the order of $\pi(0.12 a)^{2} \times(0.8 a)=0.036 a^{3}$, and the plasma energy density at the pinch moment is proportional to $E / V_{P} \sim 28 E / a^{3}$. In table 3 the parameter $28 E / a^{3}$ is listed for various PF devices and his value is of the order of $(1-10) \cdot 10^{10} \mathrm{~J} / \mathrm{m}^{3}$ [26]. The value of this energy density parameter in the case of the very small devices PF-400J and PF-50J is $(5-7) \cdot 10^{10} \mathrm{~J} / \mathrm{m}^{3}$.

Other relevant parameter in plasma foci is the called drive parameter $\left(I_{0} / a p^{1 / 2}\right)$ [20], where $I_{0}$ is the peak current, $a$ the anode radius, and $p$ the gas filling pressure for the maximum neutron yield. This drive parameter $\left(I_{0} / a p^{1 / 2}\right)$ is related with the velocity of the axial and radial phase of the plasma motion (of the order of $(0.8-1) \cdot 10^{5}$ and $(2-2.5) \cdot 10^{5} \mathrm{~m} / \mathrm{s}$ respectively for a wide range of plasma focus sizes). In fact the axial and radial velocity are proportional to $\left(I_{0} / a p^{1 / 2}\right)[4,14,20]$. For devices over the range $3 k J-1 M J$ the drive parameter $I_{0} / a p^{1 / 2}=77 \pm 7$ $k A / c m \cdot \operatorname{mbar}^{1 / 2}$ [20]. In Table 3 the drive parameter $\left(I_{0} / a p^{1 / 2}\right)$ is listed for various PF devices. The drive parameter for the very small devices PF-400J and PF-50J is evaluated in $\sim 70 \mathrm{kA} / \mathrm{cm} \cdot \mathrm{mbar}^{1 / 2}$. 
TABLE 1. The principal parameters of the plasma focus devices at the Comisión Chilena de Energía Nuclear are listed. $(*)$ Equivalent capacity of the SPEED generators.

\begin{tabular}{|l|c|c|c|c|}
\hline Device & PF-50J & PF-400J & SPEED4 & SPEED2 \\
\hline capacity $(\mu F)$ & 0.160 & 0.880 & $1.25^{*}$ & $4.16^{*}$ \\
\hline Charging voltage $(k V)$ & 35 & 35 & 100 & 300 \\
Maximum & $25-30$ & 30 & 60 & 180 \\
Typical operation & 38 & 38 & 40 & 20 \\
\hline Inductance $(n H)$ & 150 & 300 & 350 & 400 \\
\hline Time to peak current $(n s)$ & & & & \\
\hline Stored energy $(k J)$ & 0.1 & 0.54 & 6.25 & 187 \\
Maximum & $0.05-0.07$ & 0.4 & 2.25 & 67 \\
Typical operation & & & & \\
\hline Peak current $(k A)$ & 70 & 168 & 550 & 4000 \\
Maximum & $50-60$ & 127 & 330 & 2400 \\
Typical operation & 0.3 & 0.6 & 1.6 & 5.4 \\
\hline Anode radius $(\mathrm{cm})$ & 1.1 & 1.3 & 4.5 & 11 \\
\hline Cathode radius $(\mathrm{cm})$ & 0.48 & 0.7 & $1-2$ & $1.5-2.5$ \\
\hline Effective anode length $(\mathrm{cm})$ & 2.4 & 2.1 & $2.7-3.9$ & 6.5 \\
\hline Insulator length $(\mathrm{cm})$ & & & \\
\hline
\end{tabular}

TABLE 2. Summary of the results to the neutron yield obtained in the experiments presented in this article. For SPEED2 also is shown the neutron yield measured in Düsseldorf [28].

\begin{tabular}{|l|c|c|c|c|}
\hline Device & $\begin{array}{c}\text { Operation } \\
\text { enrergy } \\
(k J)\end{array}$ & $\begin{array}{c}\text { Operation } \\
\text { Pressure } \\
(m b a r)\end{array}$ & $\begin{array}{c}\text { Peak } \\
\text { Current } \\
(k A)\end{array}$ & $\begin{array}{c}\text { Neutron Yield } \\
\text { (neutrons) }\end{array}$ \\
\hline SPEED 2 & 67 & $2-3$ & 2400 & $\begin{array}{c}10^{11}-10^{12} \text { (Düsseldorf) [28] } \\
10^{10}-10^{11} \text { (CCHEN) }\end{array}$ \\
\hline SPEED 4 & 1.56 & $2-9$ & 280 & - \\
\hline PF-400J & 0.4 & 9 & 127 & $(1.06 \pm 0.13) \cdot 10^{6}$ \\
\hline PF-50 & 0.07 & 9 & 60 & $(3.3 \pm 1.6) \cdot 10^{4}$ \\
& 0.05 & 6 & 50 & $(1.1 \pm 0.5) \cdot 10^{4}$ \\
\hline
\end{tabular}

TABLE 3. The density energy parameter $28 E / a^{3}$ and the drive parameter $\left(I_{0} / a p^{1 / 2}\right)$ are listed for various PF devices, $E$ is the stored energy in the capacitor bank, $I_{0}$ is the peak cirrent, $a$ the anodo radius, and $p$ the gas filling pressure for the maximum neutron yield. The density energy parameter has a value of the order of $(1-10) \cdot 10^{10} \mathrm{~J} / \mathrm{m}^{3}$ for all the machines listed. The drive parameter has practically the same value for all the machines listed $\left(70-80 \mathrm{kA} / \mathrm{cm} \cdot \mathrm{mbar}^{1 / 2}\right)$ with the exception of the SPEED2.

\begin{tabular}{|l|c|c|c|c|c|c|}
\hline $\begin{array}{l}\text { Device } \\
\text { [reference] }\end{array}$ & $\begin{array}{c}\text { Energy } \\
E(k J)\end{array}$ & $\begin{array}{c}\text { Anode } \\
\text { radius } \\
a(\mathrm{~cm})\end{array}$ & $\begin{array}{c}\text { Peak } \\
\text { current } \\
(k A)\end{array}$ & $\begin{array}{c}\text { Pressure } \\
(\text { mbar })\end{array}$ & $\begin{array}{c}\text { Energy density } \\
\text { parameter } \\
28 E / a^{3}\left(\mathrm{~J} / \mathrm{m}^{3}\right)\end{array}$ & $\begin{array}{c}\text { Driven factor } \\
I / p^{1 / 2} a \\
k A / \mathrm{mbar}^{1 / 2} \mathrm{~cm}\end{array}$ \\
\hline PF-1000[3] & 1000 & 11.5 & 2 & 6 & $1.8 \cdot 10^{10}$ & 72 \\
\hline PF-360 [29] & 60 & 5 & 750 & 4 & $1.3 \cdot 10^{10}$ & 75 \\
\hline SPEED2 [2] & 70 & 5.4 & 2400 & $2-3$ & $1.2 \cdot 10^{10}$ & $317-259$ \\
\hline GN1 [12] & 4.7 & 1.9 & - & - & $1.9 \cdot 10^{10}$ & - \\
\hline UNU/ICTP- & 2.9 & 0.95 & 172 & 8.5 & $9.5 \cdot 10^{10}$ & 81 \\
PFF [4] & & & & & & 70 \\
\hline PF-400J [9] & 0.4 & 0.6 & 127 & 9 & $5.2 \cdot 10^{10}$ & 66.7 \\
\hline PF-50J [26] & 0.07 & 0.3 & 60 & 9 & $7.3 \cdot 10^{10}$ & 68 \\
& 0.05 & 0.3 & 50 & 6 & $5.2 \cdot 10^{10}$ & \\
\hline
\end{tabular}


It is important to note that the parameters used in the design, construction and operation of the plasma focus at SPEED2 give a drive parameter very high in comparison with the other devices listed in the table 3 . It is remarkable that if this parameter is experimentally increased over $90 \mathrm{kA} / \mathrm{cm} \cdot \mathrm{mbar}^{1 / 2}$ (modifying $I_{0}, a$, or $p$ ) the plasma focus does not work, this is the experience in the most of plasma focus [21]. However, in SPEED2 evidence of pinch has been observed and neutron yield has been measured with a high value of $\left(I_{0} / a p^{1 / 2}\right)$. An exhaustive characterization of the neutron emission from the plasma focus at SPEED2 is required to clarify this point.

The total neutron yield $Y$, is $Y=Y_{t h}+Y_{b-t}$, where $Y_{t h}$ is the thermonuclear component and $Y_{b-t}$ is the beam target component. Moo et al [22] have shown that $Y_{t h}: Y_{b-t}$ is of the order of $15: 85$ in conventional plasma focus machines.

The possibility to enhance the thermonuclear component of the neutron yield increasing the drive parameter $\left(I_{0} / a p^{1 / 2}\right)$ has been discussed by S. Lee and A. Serban [23, 24]. Lee and Serban have proposed, as the square root of the pressure, $p^{1 / 2}$, varies very little (less than $40 \%$ ) relative to the variation of $I$ (10 times or more), $(I / a)$ may by considered to have the almost a fixed value for a wide range of devices. The pinch plasma in the range of $3 k J$ to $M J$ all having practically the same temperature, $T \sim 1 \mathrm{keV}$, and density, $n \sim 10^{25} \mathrm{~m}^{-3}$, the thermonuclear component of the neutron yield will thus be proportional to $a^{4}$ ( $a^{3}$ from the volume dependence and another factor $a$ from the pinch lifetime dependence). This gives the well-known law $Y_{t h} \propto I^{4}$ that is observed in plasma focus devices. The above argument also suggests that this observed law is due to the fixed speed (or fixed energy density) operation over the whole range of plasma focus machines. If the value of $(I / a)$ is increased, speed will increase with $(I / a)$, and $T$ will increase with $(I / a)^{2}$. In the present range of operation of plasma focus $\left(\sim 1 \mathrm{keV}\right.$ when is used $\left.D_{2}\right)$, increase in $T$ will lead to increase in fusion cross section $\langle\sigma v\rangle$ proportional to $T^{\nu}$, with $\nu \sim 4$, thus $\langle\sigma v\rangle \sim(I / a)^{8}$. Thus $Y_{t h} \sim\langle\sigma v\rangle$ (volume $)($ pinch lifetime $) \propto(I / a)^{8}\left(a^{3}\right)(a)=(I / a)^{4} I^{4}$. Thus, if $(I / a)$ is a constant, this reduces to $Y_{t h} \propto I^{4}$ as noted above. Otherwise $(I / a)^{4} I^{4}$ (or $v^{4} I^{4}$ ), if (a) is kept constant as $I$ is increased then $Y_{t h} \propto I^{8}$. With this improved or enhanced yield dependence, the thermonuclear component of neutron yield will rapidly outstrip the beam target component. Assuming a simple model for the beam target component of the neutron yield, the relation $Y_{b-t} \propto I^{9 / 2} / v^{3 / 2}$ $\propto I^{9 / 2} /(I / a)^{3 / 2}$ was obtained in the reference [23]. Thus in the limit, if $a$ is keeping constant whilst increasing $I$, $Y_{b-t} \propto I^{3}$ and $Y_{t h} \propto I^{8}$. In reference [23] as way to obtain a plasma focus operating with a high value of the drive parameter a composite anode was used (a one piece anode with diameter reduced at the end was used), and a enhanced of the order of 12 to $16 \%$ in the neutron yield was reported.

In the above frame the evidence of pinch and measured neutron yield obtained in the SPEED2 with a high value of $\left(I_{0} / a p^{1 / 2}\right)$ deserve an exhaustive study and characterization of the neutron emission. On the other hand from our experiments in plasma foci with hundred and tens of joules (PF-400J and PF-50J in table 2 it is observed that the total neutron yield scaling as $Y \sim 7.73 \cdot 10^{-5} I^{4.82}$ (with $I$ in $k A$ ). This last observation motives future experiments in order to determinate the contribution of $Y_{t h}$ and $Y_{b-t}$ to the total neutron yield and to corroborate this preliminary scaling law for the region of hundred and tens of joules.

In the idea to scale a plasma focus to very low energy our question is, how low can we go in loading energy and still obtaining the plasma pinch and neutron emission? When do the plasma surface effects start to be relevant? Could these effects be favorable in order to increase the plasma energy density for much smaller devices improving the generation of fusion reactions and radiation? An extremely small device has been recently designed and constructed by us, a "nanofocus" ( $5 \mathrm{nF}, 10 \mathrm{kV}, 10 \mathrm{k}$ A, 0.25J, 10ns time to peak current, $d I / d t \sim 10^{12} \mathrm{~A} / \mathrm{s}$ ) and is currently being characterized.

The future diagnostics program in our devices considers time integrated and time resolution X-ray images, PIN diode and plastic scintillator with photomultiplier, X-ray spectroscopy and pulsed interferometry. Also a characterization of the isotropic and anisotropic components of the neutron yield will be developed [25].

\section{Acknowledgments}

This work was initially funded by a Presidential Chair in Sciences "Plasma Physics in Small Devices" awarded to L. Soto by the Chilean Government (1999-2002), and currently is partially funded by the FONDECYT grant 1030062, Chile. This work is also part of the Scientific Cooperation Project "Innovative applications of thermonuclear fusion", between Comisión Chilena de Energía Nuclear, (CCHEN, Chile) and Comisión Nacional de Energía Atómica (CNEA, Argentina). The authors appreciate the comments of Dr. Lipo Birstein of CCHEN and Dr. A. Clausse of CNEA.

\section{References}

[1] P. Silva and M. Favre, Journal of Physics D: Applied Physics 35, 2543 (2002).

[2] G. Decker, W. Kies, M. Mälzig, C. Van Valker, and G. Ziethen, Nucl. Instrum. and Methods, A249, 477 (1986).

[3] M. Scholz, L. Karpinski, M. Paduch, K. Tomaszewsky, R. Miklaszewsky, and A. Szydlowski, Nukleonika 46, 35 (2001).

[4] S. Lee, T. V. Tou, S. P. Moo, M. A. Eissa, A. V. Golap, K. H. Kewk, S. Mulyodrone, A. J. Smith, Suryad, W. Usada, and M. Zakaullah, Amer. J. Phys. 56, 62 (1988).

[5] M. Zakaullah, K. Alamgir, M. Shafiq, S. M. Hassan, M. Sharif, S. Hussain, and A. Waheed, Plasma Sources Sci. and Technol 11, 377 (2002).

[6] A. V. Dubrovsky, V. A. Gribkov, Yu. P. Ivanov, P. Lee, S. Lee, M. Lieu, and V. A. Samarain, Nukleonika 46 S1, S107 (2001).

[7] V. A. Gribkov (private comunication). 
[8] L. Soto, A. Esaulov, J. Moreno, P. Silva, G. Sylvester, M. Zambra, A. Nazarenko, and A. Clausse, Physics of Plasma 8, 2572 (2001).

[9] P. Silva, L. Soto, J. Moreno, G. Sylvester, M. Zambra, L. Altamirano, H. Bruzzone, A. Clausse, and C. Moreno, Rev. Sci. Instrum. 73, 2583 (2002).

[10] J. Moreno, P. Silva, and L. Soto, Plasma Sources Sci. and Technol. 12, 39 (2003).

[11] P. Silva, J. Moreno, L. Soto, L. Birstein, R. Mayer, and W. Kies, App. Phys. Lett. 83, 3269 (2003).

[12] C. Moreno, H. Bruzzone, J. Martinez, and A. Clausse, IEEE Trans. Plasma Sci. 28, 1735 (2000).

[13] "Measurement of low yield neutron pulses from D-D fusion reactions using a $3 \mathrm{He}$ proportional counter", J. Moreno, L. Birstein, R. E. Mayer, P. Silva, and L. Soto, submitted for publication.

[14] S. Lee, Laser and Plasma Technology, edited by S. Lee, B. C. Tan, C. S. Wong, and A. C. Chew (World Scientific, Singapore, 1985).

[15] L. Soto, H. Chuaqui, M. Favre, and E. Wyndham, Phys. Rev. Lett. 72, 2891 (1994).

[16] L. Soto, H. Chuaqui, M. Favre, R. Saavedra, E. Wyndham, M. Skowronek, P. Romeas, R. Aliaga-Rossel, and I. Mitchell, IEEE. Trans. Plasma Science 26, 1179 (1998).

[17] A. Esaulov, P. Sasorov, L. Soto, and M. Zambra, Physics of Plasma 8, 1395 (2001).

[18] L. Soto and A. Clausse, Physica Scripta 67, 77 (2003).
[19] G. F. Knoll, Radiation detection and measurements (John Wiley and Sons, 2000).

[20] S. Lee and A. Serban, IEEE Trans. Plasma Science 24, 1101 (1996).

[21] S. Lee (private communication, Cairo, Egypt, October 2003)

[22] S. P. Moo, C. K. Chakrabarty, and S. Lee, IEEE Trans. Plasma Science 19, 515 (1991).

[23] S. Lee and A. Serban, Proceedings of the 1994 International Confernece on Plasma Physics combined with the VI Latin American on Plasma Physics, vol. 1, p. 181 (1994).

[24] S. Lee, in Abstracts of the First Cairo Conference on Plasma Physics and Applications, p. 5 (Cairo, Egypt, October 2003)

[25] F. Castillo, J. J. E. Herrera, J. Rangel, M. Milanese, R. Moroso, J. Pouzo, J. I. Golzarri, and G. Espinosa, Plasma Phys. and Control. Fusion 45, 289 (2003)

[26] P. Silva, L. Soto, W. Kies and J. Moreno, Plasma Sources Sci. and Technol. 13, 329 (2004).

[27] "Demonstration of neutron production from a deuterium plasma pinch driven by a capacitor bank charged at only tens of joules". L. Soto, P. Silva, J. Moreno, M. Zambra, W. Kies, R. E. Mayer, H. Bruzzone, L. Altamirano, L. Huerta, and A. Clausse, submitted for publication.

[28] W. Kies (private communication).

[29] S. Czekaj, A. Kasperzuk, R. Miklaszewski, M. Paduch, T. Pisarczyk, and Z. Wereszczynski, Plasma Phys. Contr. Fusion 31, 587 (1989). 